\title{
Optimal Growth Strategy under Dynamic Threshold
}

\author{
CUONG LE VAN \\ IPAG Business School, CNRS, PSE, VCREME \\ ÇAĞRı SAĞLAM \\ Bilkent University \\ AGAH TURAN \\ Bilkent University
}

\begin{abstract}
We consider an economy in which the technology exhibits nonconvexities due to fixed costs associated with production. Taking into account the incentives for investment to decrease fixed costs, we characterize the circumstances under which an underdeveloped economy can catch up with the developing ones. We show that it is optimal to get rid of the fixed costs inherent in production in finite time provided that the initial level of fixed costs are not too high and the technology for reducing fixed costs is sufficiently efficient. Indeed, we obtain that even though the income disparities may be very persistent and can be perceived as poverty traps, economies with not very high initial fixed costs and sufficiently efficient technology for reducing fixed costs would ultimately converge to the same steady state level of per capita income.
\end{abstract}

\section{Introduction}

Consider an economy in which the technology exhibits nonconvexities due to fixed costs associated with production. According to Dechert and Nishimura (1983) and its extensions (e.g., Mitra and Ray 1984; Kamihigashi and Roy 2007; Hung, Le Van, and Michel 2009; Akao, Kamihigashi, and Nishimura 2011), such an economy can fall into a poverty trap if its initial capital or income falls short of the fixed cost inherent in production. However, to what extent these analyses are robust to the considerations of incentives for investment to decrease the fixed costs in production, still remains unanswered: Can such an underdeveloped economy eventually catch up with the developing ones if endowed with a technology to reduce the fixed costs? If so, how and how long will it take? If not, why not? To account for these seminal questions we consider a nonclassical optimal growth model which takes the incentives for investment to decrease fixed costs explicitly into account.

Fixed costs associated with production stem mainly from the lack of core infrastructure such as road, rail, power supply, telecommunications, irrigation, sanitation, and

Cuong Le Van, IPAG Business School, CNRS, PSE, VCREME, France (Cuong.Le-Van@univ-paris1.fr). Cağrı Sağlam, Department of Economics, Bilkent University, Ankara, Turkey (csaglam@bilkent.edu.tr). Agah Turan, Department of Economics, Bilkent University, Ankara, Turkey (agah@bilkent.edu.tr).

Received July 26, 2014; Accepted April 20, 2015.

(c) 2016 Wiley Periodicals, Inc.

Journal of Public Economic Theory, 18 (6), 2016, pp. 979-991. 
poor access to productive assets. In particular, the lack of infrastructure and the externalities arising from them have been shown to play a primary role for possible lock-ins to underdevelopment or poverty (see Rosegrant et al. 2006). For instance, in Ethiopia, the average road density is $27 \mathrm{~km} / 1000 \mathrm{~km}^{2}$, and the mean travel time to the nearest main output market is about 7 hours. Grain prices received at the farm gate are $30 \%-70 \%$ less than the market prices in the nearby main markets and about $50 \%-60 \%$ of potential revenue is lost due to inaccurate price information (see, for the details, Demeke et al. 2004; Hanjra, Ferede, and Gutta 2009). Yoshino (2008), for example, stresses that the average number of days per year for which the firms experience disruptions in electricity has an adverse effect on development in sub-Saharan Africa. These facts (see World Bank 1994, for a broad survey of the effects of the lack of infrastructure) further highlight the essence of the present analysis to a greater extent.

In this paper, we analyze the optimal growth strategy of such economies in which the fixed costs associated with production due to lack of infrastructure entail a threshold level of capital stock above which the capital stock turns out to be productive. However, in contrast with the earlier optimal growth models with nonconvex technology (see Azariadis and Stachurski 2005, for a recent survey), this threshold level of capital stock induced by the lack of infrastructure is not assumed to be exogenous and fixed. Indeed, we put the emphasis on the ability to reduce fixed costs in production and characterize the circumstances under which an underdeveloped economy can catch up with the developing ones.

We show that if it turns out to be optimal not to devote any resource to reduce the fixed costs at a certain period, then it will never be optimal to do so from that period onwards. However, if it turns out to be optimal to decrease the fixed costs in production at one period, then the accumulated capital stock will exceed the level of fixed costs sooner or later. Indeed, under mild conditions on the efficiency of the infrastructure technology and the initial level of fixed costs, we prove that it is optimal to get rid of the fixed costs inherent in production at a finite period of time so that the economy will converge to a positive steady state level of physical capital independent of its initial level. Put differently, we show how the threshold dynamics prediction of the nonclassical optimal growth models (e.g., Dechert and Nishimura 1983) can be overturned by taking into account the incentives to reduce the threshold level of capital stock stemming from the fixed costs associated with production. We indicate that even though the income disparities may be very persistent and can be perceived as poverty traps, all economies with not very high initial fixed costs and sufficiently efficient technology to reduce them would ultimately converge to the same steady state level of per capita income.

On empirical grounds, our results provide a link between the recent estimates of Kremer, Onatski, and Stock (2001) and that of Quah $(1996,1997)$. Quah's results have supported a bimodal distribution of per capita income across countries. On the contrary, Kremer et al. (2001) and Jones (1997) have questioned the robustness of the lower peak in output and argued that the long-run income distribution is unimodal but the bimodality appears during the transition. Moreover, Feyrer (2008) has noted that the twin peaked income distribution in Quah $(1996,1997)$ appear to be driven by twin peaks in productivity and has argued empirically that the low peak in productivity may be a transitory phenomenon (see also Azariadis and Stachurski 2004; Kraay and Raddatz 2007). Actually, the delicacy in this controversy regarding the bimodal income distribution reduces to produce the mechanisms under which the twin-peaked transitional dynamics eventually converge to a "mass point in the cross-section distribution" (see Quah 2001). In this respect, our analysis fulfills this need by putting forward the incentives to reduce the fixed costs associated with production in an optimal growth model with 
nonconvex technology and suggests that the dynamic interaction between capital accumulation and fixed costs that impede productivity may act in the long run to eliminate the low peak in productivity.

The rest of the paper is organized as follows. Section 2 describes the model and Section 3 provides the dynamic properties of optimal paths. Section 4 concludes.

\section{Model}

We consider an optimal growth model which takes into account the incentives for investment to decrease fixed costs in production explicitly. At each date $t \in \mathbb{Z}_{+}$, a single good is produced according to the technology,

$$
\tilde{f}\left(k_{t} \mid \bar{k}_{t}\right)= \begin{cases}f\left(k_{t}-\bar{k}_{t}\right), & \text { if } k_{t} \geq \bar{k}_{t}, \\ 0, & \text { if } k_{t}<\bar{k}_{t},\end{cases}
$$

in which the labor input is supposed to be constant and normalized to 1 . Here $\bar{k}_{t}$ is the threshold level of capital stock that represents the fixed cost in production due to corruption or the lack of infrastructure which may include roads, power, irrigation, or energy, and $k_{t}$ is the stock of physical capital at the beginning of period $t$. The depreciation rate is $\delta \in(0,1)$. At each period current output must be divided between current consumption, $c_{t}$, gross investment in physical capital, $i_{t}$, and the expenditures for reducing the fixed costs of production (i.e., investment on infrastructure), $m_{t}$. The infrastructure technology is given by a function $\varphi$ so that $\bar{k}_{t+1}=\bar{k}_{t}-\varphi\left(m_{t}\right)$.

In this economy, the social utility is represented by $\sum_{t=0}^{+\infty} \beta^{t} u\left(c_{t}\right)$, where $\beta \in(0,1)$ is the discount factor. The optimal growth problem can then be formalized as follows:

$$
\max _{\left\{c_{t}, m_{t}, k_{t+1}, \bar{k}_{t+1}\right\}_{t=0}^{\infty}} \sum_{t=0}^{\infty} \beta^{t} u\left(c_{t}\right),
$$

subject to

$$
\begin{aligned}
\forall t, c_{t}+m_{t}+i_{t} & \leq \tilde{f}\left(k_{t}, \bar{k}_{t}\right), \\
k_{t+1} & =i_{t}+(1-\delta) k_{t}, \\
\bar{k}_{t+1} & =\bar{k}_{t}-\varphi\left(m_{t}\right), \\
c_{t} & \geq 0, m_{t} \geq 0, k_{t} \geq 0, \bar{k}_{t} \geq 0, \\
\bar{k}_{0} & >0, k_{0}>0 \text { are given. }
\end{aligned}
$$

We maintain the following assumptions throughout the paper.

ASSUMPTION 1: $u: \mathbb{R}_{+} \rightarrow \mathbb{R}_{+}$is twice continuously differentiable and satisfies $u(0)=0$, $u^{\prime}>0, u^{\prime \prime}<0, u^{\prime}(0)=+\infty$.

ASSUMPTION 2: $f: \mathbb{R}_{+} \rightarrow \mathbb{R}_{+}$is twice continuously differentiable and satisfies $f(0)=0$, $f^{\prime}>0, f^{\prime \prime}<0, f^{\prime}(0)=+\infty, \lim _{x \rightarrow+\infty} f^{\prime}(x)<\delta$.

ASSUMPTION 3: $\varphi: \mathbb{R}_{+} \rightarrow \mathbb{R}_{+}$is twice continuously differentiable and satisfies $\varphi(0)=0$, $\varphi^{\prime}>0, \varphi^{\prime \prime}<0, \varphi^{\prime}(0)>1$.

ASSUMPTION 4: $k_{0} \neq \bar{k}_{0}$. 
Note from Assumption 2 that when $k \neq \bar{k}, \tilde{f}_{k}^{\prime}=-\tilde{f}_{\bar{k}}^{\prime}$. Let $g(x)=\varphi^{-1}(x), \forall x \geq 0$. Problem $\mathcal{P}$ is actually equivalent to

$$
\max _{\left\{c_{t}, k_{t+1}, \bar{k}_{t+1}\right\}_{t=0}^{\infty}} \sum_{t=0}^{\infty} \beta^{t} u\left(c_{t}\right),
$$

subject to

$$
\begin{aligned}
c_{t}+k_{t+1}+g\left(\bar{k}_{t}-\bar{k}_{t+1}\right) & \leq \tilde{f}\left(k_{t}, \bar{k}_{t}\right)+(1-\delta) k_{t}, \\
0 & \leq \bar{k}_{t+1} \leq \bar{k}_{t}, \\
k_{t} & \geq 0, c_{t} \geq 0, \\
\bar{k}_{0} & >0, k_{0}>0 \text { are given. }
\end{aligned}
$$

We adopt the following standard definitions and notations. An infinite sequence $\left\{x_{t}\right\}_{t=0}^{\infty}$ will be denoted by $\boldsymbol{x}$. We say that the sequences $\boldsymbol{c}, \boldsymbol{k}, \overline{\boldsymbol{k}}$ are feasible from $k_{0}$ and $\bar{k}_{0}$ if they satisfy the constraints of Problem $\mathcal{P}^{\prime}$. A stationary path is a constant path. A capital stock $k \geq 0$ and a fixed cost $\bar{k} \geq 0$ constitute a steady state if the associated stationary path is optimal.

\section{Properties of Optimal Paths}

In this section, we present various preliminary results on the properties of optimal paths that will prove to be useful in presenting our main result.

PROPOSITION 1: For any $\left(k_{0}, \bar{k}_{0}\right)$, there exists an optimal path $(\boldsymbol{c}, \boldsymbol{k}, \overline{\boldsymbol{k}})$ which satisfies

$$
\forall t, 0 \leq k_{t} \leq M=\max \left[k_{0}, \tilde{k}\right], 0 \leq c_{t} \leq f(M),
$$

where $f(\tilde{k})=\delta \tilde{k}$.

Proof: See, e.g., Le Van and Morhaim (2002), Theorem 1.

LEMMA 1: An optimal path $(\boldsymbol{c}, \boldsymbol{k}, \overline{\boldsymbol{k}})$ from $\left(k_{0}, \bar{k}_{0}\right)$ satisfies

$$
\forall t, c_{t}>0, k_{t}>0 .
$$

Proof: Since $u$ is strictly increasing the feasible consumption path $(0,0, \ldots, 0 \ldots)$ cannot be optimal. Indeed, the path $(\boldsymbol{c}, \boldsymbol{k}, \overline{\boldsymbol{k}})$ defined by

$$
\begin{aligned}
& \bar{k}_{t}=\bar{k}_{0}, k_{t}=0, \quad c_{t}=0, \forall t \geq 1, \\
& c_{0}=\tilde{f}\left(k_{0}, \bar{k}_{0}\right)+(1-\delta) k_{0}>0,
\end{aligned}
$$

is feasible and the utility obtained with this sequence is strictly positive. Hence, there exists some $t$ such that $c_{t}>0$. Without loss of generality, assume $c_{0}=0$ and $c_{1}>0$. We have $k_{1}>0$ and $c_{1}+k_{2}+g\left(\bar{k}_{1}-\bar{k}_{2}\right)=\tilde{f}\left(k_{1}, \bar{k}_{1}\right)+(1-\delta) k_{1}$.

Consider first that $k_{1}>\bar{k}_{1}$. Choose some $\varepsilon>0$ such that $k_{1}-\varepsilon>\bar{k}_{1} \geq 0$ and $f\left(k_{1}-\right.$ $\left.\varepsilon-\bar{k}_{1}\right)+(1-\delta)\left(k_{1}-\varepsilon\right)-g\left(\bar{k}_{1}-\bar{k}_{2}\right)-k_{2}>0$. Define sequences $\left(\boldsymbol{c}^{\prime}, \boldsymbol{k}^{\prime}\right)$ by

$$
c_{0}^{\prime}=\varepsilon, c_{1}^{\prime}=f\left(k_{1}-\varepsilon-\bar{k}_{1}\right)+(1-\delta)\left(k_{1}-\varepsilon\right)-g\left(\bar{k}_{1}-\bar{k}_{2}\right)-k_{2},
$$

and

$$
\forall t \geq 2, \quad c_{t}^{\prime}=c_{t}, k_{t}^{\prime}=k_{t}
$$


These sequences are feasible from $\left(k_{0}, \bar{k}_{0}\right)$. We obtain that

$$
\begin{aligned}
\Delta(\varepsilon) & =\sum_{t=0}^{\infty} \beta^{t} u\left(c_{t}^{\prime}\right)-\sum_{t=0}^{\infty} \beta^{t} u\left(c_{t}\right) \\
& =u\left(c_{0}^{\prime}\right)+\beta u\left(c_{1}^{\prime}\right)-u\left(c_{0}\right)-\beta u\left(c_{1}\right) \\
& \geq \varepsilon\left[u^{\prime}\left(c_{0}^{\prime}\right)-\beta u^{\prime}\left(c_{1}^{\prime}\right)\left(\frac{f\left(k_{1}-\bar{k}_{1}\right)-f\left(k_{1}-\varepsilon-\bar{k}_{1}\right)}{\varepsilon}+(1-\delta)\right)\right] .
\end{aligned}
$$

Note that $\lim _{\varepsilon \rightarrow 0} u^{\prime}\left(c_{0}^{\prime}\right)=+\infty$ and $\lim _{\varepsilon \rightarrow 0} u^{\prime}\left(c_{1}^{\prime}\right)\left(\frac{f\left(k_{1}-\bar{k}_{1}\right)-f\left(k_{1}-\varepsilon-\bar{k}_{1}\right)}{\varepsilon}+(1-\delta)\right)<+\infty$. Hence, $\Delta(\varepsilon)>0$ when $\varepsilon$ is small enough, a contradiction.

Consider now that $k_{1} \leq \bar{k}_{1}$. We have $k_{1}>0$ and

$$
\begin{aligned}
\left(c_{0}+\varepsilon\right)+\left(k_{1}-\varepsilon\right)+g\left(\bar{k}_{0}-\bar{k}_{1}\right) & =\tilde{f}\left(k_{0}, \bar{k}_{0}\right)+(1-\delta) k_{0} \\
\left(c_{1}-(1-\delta) \varepsilon\right)+k_{2}+g\left(\bar{k}_{1}-\bar{k}_{2}\right) & =\tilde{f}\left(k_{1}-\varepsilon, \bar{k}_{1}\right)+(1-\delta)\left(k_{1}-\varepsilon\right)
\end{aligned}
$$

since $0=\tilde{f}\left(k_{1}-\varepsilon, \bar{k}_{1}\right)=\tilde{f}\left(k_{1}, \bar{k}_{1}\right)$. Then

$$
\begin{aligned}
\Delta(\varepsilon) & =u(\varepsilon)-u(0)+\beta\left[u\left(c_{1}-(1-\delta) \varepsilon\right)-u\left(c_{1}\right)\right. \\
& \geq u^{\prime}(\varepsilon) \varepsilon-\beta u^{\prime}\left(c_{1}-(1-\delta) \varepsilon\right)(1-\delta) \varepsilon .
\end{aligned}
$$

We obtain $\Delta(\varepsilon) / \varepsilon>0$ when $\varepsilon$ is small enough.

Now we claim that $k_{1}>0$. Assume on the contrary that $k_{1}=0$. In this case, we have $k_{t}=0, c_{t}=0$ and $m_{t}=0$ so that $\bar{k}_{t}=\bar{k}_{1}, \forall t \geq 1$. Moreover, $\bar{k}_{1}=\bar{k}_{0}$ as utility is increasing in consumption. Choose some $\varepsilon>0$ such that $c_{0}-\varepsilon>0$. Consider sequences $\left(\boldsymbol{c}^{\prime}, \boldsymbol{k}^{\prime}\right)$ where

$$
c_{0}^{\prime}=c_{0}-\varepsilon, k_{1}^{\prime}=\varepsilon, c_{1}^{\prime}=\tilde{f}\left(k_{1}^{\prime}, \bar{k}_{1}\right)+(1-\delta) \varepsilon,
$$

and

$$
\forall t \geq 2, \quad c_{t}^{\prime}=c_{t}, k_{t}^{\prime}=k_{t}
$$

We compute that

$$
\begin{aligned}
\Delta(\varepsilon) & =u\left(c_{0}-\varepsilon\right)+\beta u\left(c_{1}^{\prime}\right)-u\left(c_{0}\right) \\
& \geq \varepsilon\left[\frac{\beta u((1-\delta) \varepsilon)}{\varepsilon}-u^{\prime}\left(c_{0}-\varepsilon\right)\right] .
\end{aligned}
$$

As $\varepsilon \rightarrow 0$, we have $\frac{\beta u((1-\delta) \varepsilon)}{\varepsilon} \rightarrow+\infty$ and $-u^{\prime}\left(c_{0}-\varepsilon\right) \rightarrow-u^{\prime}\left(c_{0}\right)>-\infty$. This implies that $\Delta(\varepsilon)>0$ when $\varepsilon$ is small enough, a contradiction. We have proved that if $c_{0}>0$ then $c_{1}>0$ and $k_{1}>0$. By induction, we have $c_{t}>0, k_{t}>0, \forall t$.

Recall that $k_{0} \neq \bar{k}_{0}$. The following lemma shows that the level of the capital stock will never be equal to the fixed cost of production along the optimal path.

LEMMA 2: Let $(\boldsymbol{c}, \boldsymbol{k}, \overline{\boldsymbol{k}})$ be an optimal path from $\left(k_{0}, \bar{k}_{0}\right)$. We have $k_{t+1} \neq \bar{k}_{t+1}, \forall t$.

Proof: Assume $k_{1}=\bar{k}_{1}$. We have

$$
\begin{aligned}
c_{0}+k_{1}+m_{0} & =\tilde{f}\left(k_{0}, \bar{k}_{0}\right)+(1-\delta) k_{0}, \\
c_{1}+k_{2}+m_{1} & =\tilde{f}\left(k_{1}, \bar{k}_{1}\right)+(1-\delta) k_{1}=(1-\delta) k_{1},
\end{aligned}
$$

where $c_{0}>0$ by Lemma 1 . 
Consider the sequences $\left(\boldsymbol{c}^{\prime}, \boldsymbol{k}^{\prime}\right)$ defined as follows:

$$
\begin{aligned}
& c_{0}^{\prime}=c_{0}-\varepsilon, \\
& k_{1}^{\prime}=k_{1}+\varepsilon, \\
& c_{1}^{\prime}=\tilde{f}\left(k_{1}+\varepsilon, \bar{k}_{1}\right)+(1-\delta)\left(k_{1}+\varepsilon\right)-k_{2}-m_{1},
\end{aligned}
$$

and

$$
\forall t \geq 2, \quad c_{t}^{\prime}=c_{t}, k_{t}^{\prime}=k_{t} .
$$

$\left(\mathbf{c}^{\prime}, \mathbf{k}^{\prime}, \overline{\mathbf{k}}\right)$ is also feasible from $\left(k_{0}, \bar{k}_{0}\right)$. We compute

$$
\begin{aligned}
\Delta(\varepsilon) & =\sum_{t=0}^{\infty} \beta^{t} u\left(c_{t}^{\prime}\right)-\sum_{t=0}^{\infty} \beta^{t} u\left(c_{t}\right) \\
& =u\left(c_{0}-\varepsilon\right)+\beta u\left(c_{1}^{\prime}\right)-u\left(c_{0}\right)-\beta u\left(c_{1}\right) \\
& \geq \varepsilon\left[-u^{\prime}\left(c_{0}-\varepsilon\right)+\beta u^{\prime}\left(c_{1}^{\prime}\right)\left(\frac{f(\varepsilon)}{\varepsilon}+(1-\delta)\right)\right] .
\end{aligned}
$$

As $\lim _{\varepsilon \rightarrow 0}\left[-u^{\prime}\left(c_{0}-\varepsilon\right)+\beta u^{\prime}\left(c_{1}^{\prime}\right)\left(\frac{f(\varepsilon)}{\varepsilon}+(1-\delta)\right)\right]=+\infty$, we obtain a contradiction. Hence, $k_{1} \neq \bar{k}_{1}$.

LEMMA 3: Let $(\boldsymbol{c}, \boldsymbol{k}, \overline{\boldsymbol{k}})$ be an optimal path from $\left(k_{0}, \bar{k}_{0}\right)$. If $\bar{k}_{T}=\bar{k}_{T+1}$ then $\bar{k}_{T}=\bar{k}_{T+t}$, $\forall t \geq 0$.

Proof: Assume without loss of generality that $\bar{k}_{1}=\bar{k}_{0}>0$. We have $m_{0}=0$ and

$$
\forall t, c_{t}+k_{t+1}+g\left(\bar{k}_{t}-\bar{k}_{t+1}\right)=\tilde{f}\left(k_{t}, \bar{k}_{t}\right)+(1-\delta) k_{t} .
$$

Suppose on the contrary that $\bar{k}_{3} \leq \bar{k}_{2}<\bar{k}_{1}$. Note that an optimal solution to $P^{\prime}$ must also be optimal over any finite period. Consider the following three-period optimization problem for a given initial condition $\left(k_{0}, \bar{k}_{0}\right)$ and terminal condition $\left(k_{3}, \bar{k}_{3}\right)$ :

$$
\begin{array}{r}
\max _{\left\{k_{1}, k_{2}, y_{1}, y_{2}\right\}} u\left(\tilde{f}\left(k_{0}-\bar{k}_{0}\right)+(1-\delta) k_{0}-k_{1}-g\left(\bar{k}_{0}-y_{1}\right)\right) \\
+\beta u\left(\tilde{f}\left(k_{1}-y_{1}\right)+(1-\delta) k_{1}-k_{2}-g\left(y_{1}-y_{2}\right)\right) \\
+\beta^{2} u\left(\tilde{f}\left(k_{2}-y_{2}\right)+(1-\delta) k_{2}-k_{3}-g\left(y_{2}-\bar{k}_{3}\right)\right)
\end{array}
$$

subject to

$$
\begin{aligned}
y_{1}-\bar{k}_{0} & \leq 0, \\
y_{2}-y_{1} & \leq 0, \\
\bar{k}_{3}-y_{2} & \leq 0, \\
k_{1} & \geq 0, \\
k_{2} & \geq 0 .
\end{aligned}
$$

The Lagrangian associated with this optimization problem can be written as

$$
\begin{aligned}
\mathcal{L}= & u\left(\tilde{f}\left(k_{0}, \bar{k}_{0}\right)+(1-\delta) k_{0}-k_{1}-g\left(\bar{k}_{0}-y_{1}\right)\right) \\
& +\beta u\left(\tilde{f}\left(k_{1}, y_{1}\right)+(1-\delta) k_{1}-k_{2}-g\left(y_{1}-y_{2}\right)\right) \\
& +\beta^{2} u\left(\tilde{f}\left(k_{2}, y_{2}\right)+(1-\delta) k_{2}-k_{3}-g\left(y_{2}-\bar{k}_{3}\right)\right) \\
& -\lambda_{1}\left(y_{1}-\bar{k}_{0}\right)-\lambda_{2}\left(y_{2}-y_{1}\right)-\lambda_{3}\left(\bar{k}_{3}-y_{2}\right)+\mu_{1} k_{1}+\mu_{2} k_{2} .
\end{aligned}
$$


Note that $k_{1}=0$ implies $c_{1}=0, k_{2}=0$ and $k_{2}=0$ furthermore implies $c_{2}=0$, which are impossible. We have already proved that $k_{1}>0, k_{2}>0$ so that $\mu_{1}=0$ and $\mu_{2}=0$. Moreover, we know that $k_{1} \neq \bar{k}_{1}$ and $k_{2} \neq \bar{k}_{2}$ (by Lemma 2). Accordingly, the first-order conditions of optimality reveal that

$$
\begin{gathered}
u^{\prime}\left(c_{0}\right)=\beta u^{\prime}\left(c_{1}\right)\left[\tilde{f}_{k}^{\prime}\left(k_{1}, \bar{k}_{1}\right)+1-\delta\right], \\
\beta u^{\prime}\left(c_{1}\right)=\beta^{2} u^{\prime}\left(c_{2}\right)\left[\tilde{f}_{k}^{\prime}\left(k_{2}, \bar{k}_{2}\right)+1-\delta\right], \\
u^{\prime}\left(c_{0}\right) g^{\prime}\left(\bar{k}_{0}-\bar{k}_{1}\right)=\beta u^{\prime}\left(c_{1}\right)\left[-\tilde{f}_{\bar{k}}^{\prime}\left(k_{1}, \bar{k}_{1}\right)+g^{\prime}\left(\bar{k}_{1}-\bar{k}_{2}\right)\right]+\lambda_{1}-\lambda_{2}, \\
\beta u^{\prime}\left(c_{1}\right) g^{\prime}\left(\bar{k}_{1}-\bar{k}_{2}\right)=\beta^{2} u^{\prime}\left(c_{2}\right)\left[-\tilde{f}_{\bar{k}}^{\prime}\left(k_{2}, \bar{k}_{2}\right)+g^{\prime}\left(\bar{k}_{2}-\bar{k}_{3}\right)\right]+\lambda_{2}-\lambda_{3},
\end{gathered}
$$

where

$$
\begin{aligned}
& \lambda_{1} \geq 0, \lambda_{1}\left(\bar{k}_{1}-\bar{k}_{0}\right)=0, \\
& \lambda_{2} \geq 0, \lambda_{2}\left(\bar{k}_{2}-\bar{k}_{1}\right)=0, \\
& \lambda_{3} \geq 0, \lambda_{3}\left(\bar{k}_{3}-\bar{k}_{2}\right)=0 .
\end{aligned}
$$

By means of (3) and (5), we obtain that

$$
\tilde{f}_{k}^{\prime}\left(k_{1}, \bar{k}_{0}\right)+1-\delta=\frac{1}{g^{\prime}(0)}\left[\tilde{f}_{k}^{\prime}\left(k_{1}, \bar{k}_{0}\right)+g^{\prime}\left(\bar{k}_{1}-\bar{k}_{2}\right)+\left(\lambda_{1}-\lambda_{2}\right)\right] .
$$

Since $g$ is increasing, it is immediate that

$$
\begin{aligned}
\frac{\lambda_{2}-\lambda_{1}}{g^{\prime}(0)} & =\left(\frac{1}{g^{\prime}(0)}-1\right) \tilde{f}_{k}^{\prime}\left(k_{1}, \bar{k}_{0}\right)+\frac{g^{\prime}\left(\bar{k}_{1}-\bar{k}_{2}\right)}{g^{\prime}(0)}-(1-\delta) \\
& \geq\left(\frac{1}{g^{\prime}(0)}-1\right) \tilde{f}_{k}^{\prime}\left(k_{1}, \bar{k}_{0}\right)+\delta .
\end{aligned}
$$

As $\bar{k}_{3} \leq \bar{k}_{2}<\bar{k}_{1}$, we get $\lambda_{2}=0$ so that

$$
0 \geq \frac{-\lambda_{1}}{g^{\prime}(0)} \geq\left(\frac{1}{g^{\prime}(0)}-1\right) \tilde{f}_{k}^{\prime}\left(k_{1}, \bar{k}_{0}\right)+\delta>0
$$

leads to a contradiction.

We have shown that if it is optimal not to devote any resources to reduce the fixed cost at a certain time period, then it will always be optimal not to do so from that period onwards. We will now demonstrate that if it is optimal to decrease the fixed cost in production at some period then the accumulated capital stock should exceed the level of fixed cost sooner or later. Put differently, if there is no incentive to make the capital stock larger than the fixed cost in production then there will be no investment to reduce the fixed cost at all.

LEMMA 4: Let $(\boldsymbol{c}, \boldsymbol{k}, \overline{\boldsymbol{k}})$ be an optimal path from $\left(k_{0}, \bar{k}_{0}\right)$. If $\overline{\boldsymbol{k}}_{T}>\bar{k}_{T+1}$ then we cannot have $k_{t}<\bar{k}_{t}, \forall t \geq T$.

Proof: Let $\bar{k}_{T}>\bar{k}_{T+1}$. We have $m_{T}>0$. Suppose on the contrary $k_{t}<\bar{k}_{t}, \forall t \geq T$, so that

$$
c_{t}+k_{t+1}+m_{t}=(1-\delta) k_{t}, \forall t \geq T .
$$


Consider $\left(\boldsymbol{c}^{\prime}, \boldsymbol{k}, \overline{\boldsymbol{k}}^{\prime}\right)$ that depart from $(\boldsymbol{c}, \boldsymbol{k}, \overline{\boldsymbol{k}})$ only by letting $c_{T}^{\prime}=c_{T}+m_{T}>c_{T}$, and $m_{T}^{\prime}=0$ so that $\bar{k}_{T+1}^{\prime}=\bar{k}_{T}$. Note that

$$
k_{T+1}<(1-\delta) k_{T}<(1-\delta) \bar{k}_{T}<\bar{k}_{T},
$$

and accordingly,

$$
\bar{k}_{T+1}^{\prime}>\bar{k}_{T+1} .
$$

Hence, $\left(\boldsymbol{c}^{\prime}, \boldsymbol{k}, \overline{\boldsymbol{k}}^{\prime}\right)$ is a feasible path from $\left(k_{0}, \bar{k}_{0}\right)$. However, as $u($.$) is strictly increasing,$ $\left(\boldsymbol{c}^{\prime}, \boldsymbol{k}, \overline{\boldsymbol{k}}^{\prime}\right)$ provides a higher social utility, a contradiction.

We now show that the fixed costs in production will monotonically converge to $\bar{k} \geq 0$ within a finite period of time.

PROPOSITION 2: Let $(\boldsymbol{c}, \boldsymbol{k}, \overline{\boldsymbol{k}})$ be an optimal path from $\left(k_{0}, \overline{\boldsymbol{k}}_{0}\right)$. Assume $\overline{\boldsymbol{k}}_{t+1}>0, \forall t \geq 0$. Then there exists $T$ such that $\bar{k}_{T}=\bar{k}_{T+t}, \forall t \geq 0$.

Proof: Suppose the statement of the proposition is false. Then, by Lemma 3, we have $\bar{k}_{t}>\bar{k}_{t+1}, \forall t$. Since $\bar{k}_{0}>\bar{k}_{1}$, by Lemma 4 , there exists $t_{0}$ such that $k_{t_{0}}>\bar{k}_{t_{0}}$. Similarly, $\bar{k}_{t_{0}}>$ $\bar{k}_{t_{0}+1}$ implies that there exists $t_{1}>t_{0}$ such that $k_{t_{1}}>\bar{k}_{t_{1}}$. Then there exists a subsequence $\left\{t_{v}\right\}$ such that $k_{t_{v}+1}>\bar{k}_{t_{v}+1}>0$ and $\bar{k}_{t_{v}}>\bar{k}_{t_{v}+1}>\bar{k}_{t_{v}+2}$. By (3) and (5), we have

$$
g^{\prime}\left(\bar{k}_{t_{v}}-\bar{k}_{t_{v}+1}\right)=\frac{f^{\prime}\left(k_{t_{v}+1}-\bar{k}_{t_{v}+1}\right)+g^{\prime}\left(\bar{k}_{t_{v}+1}-\bar{k}_{t_{v}+2}\right)}{f^{\prime}\left(k_{t_{v}+1}-\bar{k}_{t_{v}+1}\right)+1-\delta} .
$$

Define $x_{t_{v}}=f^{\prime}\left(k_{t_{v}+1}-\bar{k}_{t_{v}+1}\right), y_{t_{v}}=g^{\prime}\left(\bar{k}_{t_{v}}-\bar{k}_{t_{v}+1}\right)$, and note that

$$
y_{t_{v}+1}=g^{\prime}\left(\bar{k}_{t_{v}+1}-\bar{k}_{t_{v}+2}\right) \rightarrow g^{\prime}(0) .
$$

By (7), we obtain

$$
x_{t_{v}}\left(y_{t_{v}}-1\right)=y_{t_{v}+1}-(1-\delta) y_{t_{v}} .
$$

Noting that

$$
0 \leq x_{t_{v}} \rightarrow \frac{\delta g^{\prime}(0)}{g^{\prime}(0)-1}<0
$$

raises a contradiction to the existence of a subsequence $\left\{t_{v}\right\}$ such that $k_{t_{v}+1}>\bar{k}_{t_{v}+1}>0$ and $\bar{k}_{t_{v}}>\bar{k}_{t_{v}+1}>\bar{k}_{t_{v}+2}$.

In accordance with Proposition 2, for $t \geq T$, the Problem $\mathcal{P}^{\prime}$ can be recast as

$$
\max _{\left\{c_{t}, k_{t+1}\right\}_{t=0}^{\infty}} \sum_{t=0}^{\infty} \beta^{t} u\left(c_{t}\right)
$$

subject to

$$
\begin{aligned}
\forall t, c_{t}+k_{t+1} & \leq \tilde{f}\left(k_{t}, \bar{k}\right)+(1-\delta) k_{t} \\
k_{t} & \geq 0, c_{t} \geq 0, \\
\bar{k}_{T} & =\bar{k} \geq 0, \\
k_{0} & =k_{T}>0, \text { given. }
\end{aligned}
$$


In what follows our aim is to prove that it is indeed optimal to get rid of the fixed costs inherent in production at a finite period of time so that $\bar{k}=0$, and that the capital stock converges to a steady state level $k^{s}$ where $f^{\prime}\left(k^{s}\right)+(1-\delta)=\frac{1}{\beta}$.

To do so, let us first define $\hat{k}(a)$ in accordance with

$$
f^{\prime}(\hat{k}(a)-a)=\max _{k>\bar{k}_{0}} \frac{f(k-a)}{k}=\frac{f(\hat{k}(a)-a)}{\hat{k}(a)} .
$$

We will now show that $\hat{k}($.$) is increasing.$

\section{LEMMA 5:}

(i) The function $\hat{k}($.$) is increasing.$

(ii) For any $a>0$, we have $a<\hat{k}(a)$.

(iii) $\hat{k}(\bar{k}) \leq \hat{k}\left(\bar{k}_{0}\right)$.

Proof:

(i) $\hat{k}(a) \quad$ solves $\quad k=\frac{f(k-a)}{f^{\prime}(k-a)}=z(k-a)$, for $k \geq a$. We have $z^{\prime}(k-a)=$ $1-\frac{f(k-a) f^{\prime \prime}(k-a)}{f^{\prime}(k-a) f^{\prime}(k-a)}>1$, by the strict concavity of $f$. When $a$ increases, the graph of $z(k-a)$ shifts to the right and $\hat{k}(a)$ increases.

(ii) We have $f^{\prime}(\hat{k}(a)-a)<f^{\prime}(0)=f^{\prime}(a-a)$. This implies $\hat{k}(a)>a$.

(iii) Since $\bar{k} \leq \bar{k}_{0}$, we have $\hat{k}(\bar{k}) \leq \hat{k}\left(\bar{k}_{0}\right)$.

Let $k^{*}$ be defined by

$$
f^{\prime}\left(k^{*}-\bar{k}\right)+1-\delta=\frac{1}{\beta}
$$

Actually, $k^{*}=k^{s}+\bar{k}$. Define $c^{*}$ as

$$
\begin{aligned}
c^{*} & =f\left(k^{*}-\bar{k}\right)+(1-\delta) k^{*}-k^{*} \\
& =f\left(k^{*}-\bar{k}\right)-\delta k^{*} \\
& =f\left(k^{s}\right)-\delta\left(k^{s}+\bar{k}\right) .
\end{aligned}
$$

ASSUMPTION 5: $f\left(k^{s}\right)-\delta\left(k^{s}+\bar{k}_{0}\right)>0$.

LEMMA 6: Under Assumption 5, we have $c^{*}>0$.

Proof: It is immediate from $\bar{k} \leq \bar{k}_{0}$.

We want to prove that the threshold will be exhausted in finite time. We proceed in two steps. Step 1 consists of proving that $\left(k^{*}, \bar{k}\right)$ is an optimal steady state (Lemma 7 below). This requires the assumption that the fixed costs in production is not too large (Assumption 6 below). Step 2 is to prove that actually the steady state $\bar{k}$ is zero (Proposition 3).

ASSUMPTION 6: $\hat{k}\left(\bar{k}_{0}\right)<k^{s}$. 
LEMMA 7: $\left(k^{*}, \bar{k}\right)$ is an optimal steady state.

Proof: Let

$$
F\left(k_{t}, \bar{k}\right)= \begin{cases}f\left(k_{t}-\bar{k}\right), & \text { if } k_{t} \geq \hat{k}(\bar{k}), \\ k_{t} f^{\prime}(\hat{k}(\bar{k})-\bar{k}), & \text { if } k_{t}<\hat{k}(\bar{k}) .\end{cases}
$$

Note that $F\left(k_{t}, \bar{k}\right)$ is strictly increasing, concave and differentiable. Consider the following optimization problem:

$$
\max _{\left\{k_{t+1}\right\}_{t=0}^{\infty}} \sum_{t=0}^{\infty} \beta^{t} u\left(F\left(k_{t}, \bar{k}\right)+(1-\delta) k_{t}-k_{t+1}\right)
$$

subject to

$$
\begin{aligned}
\forall t, 0 & \leq k_{t+1} \leq F\left(k_{t}, \bar{k}\right)+(1-\delta) k_{t}, \\
k_{t} & \geq 0, \\
\bar{k} & \geq 0, k_{0}>0 \text { are given. }
\end{aligned}
$$

By construction, it is immediate that $k^{*}$ is an optimal steady state of Problem $\mathcal{Q}$.

Let $(\boldsymbol{k}, \overline{\boldsymbol{k}})$ be a feasible path from $\left(k^{*}, \bar{k}_{0}\right)$ for the original problem $\mathcal{P}^{\prime}$. Recall that Assumption 6 implies $\hat{k}\left(\bar{k}_{0}\right)<k^{s}+\bar{k}=k^{*}$.

We have

$$
\begin{aligned}
c_{0}+k_{1}+g\left(\bar{k}_{0}-\bar{k}_{1}\right) & \leq \tilde{f}\left(k^{*}, \bar{k}_{0}\right)+(1-\delta) k_{0}, \\
c_{1}+k_{2}+g\left(\bar{k}_{1}-\bar{k}_{2}\right) & \leq \tilde{f}\left(k_{1}, \bar{k}_{1}\right)+(1-\delta) k_{1}, \\
& \vdots \\
c_{t}+k_{t+1}+g\left(\bar{k}_{t}-\bar{k}_{t+1}\right) & \leq \tilde{f}\left(k_{t}, \bar{k}_{t}\right)+(1-\delta) k_{t},
\end{aligned}
$$

so that

$$
\begin{aligned}
c_{0}+k_{1} \leq & \tilde{f}\left(k^{*}, \bar{k}\right)+(1-\delta) k_{0} \leq F\left(k^{*}, \bar{k}\right)+(1-\delta) k_{0}, \\
c_{1}+k_{2} \leq & \tilde{f}\left(k_{1}, \bar{k}\right)+(1-\delta) k_{1} \leq F\left(k_{1}, \bar{k}\right)+(1-\delta) k_{1}, \\
& \vdots \\
c_{t}+k_{t+1} \leq & \tilde{f}\left(k_{t}, \bar{k}\right)+(1-\delta) k_{t} \leq F\left(k_{t}, \bar{k}\right)+(1-\delta) k_{t},
\end{aligned}
$$

since $\bar{k}_{0} \geq \bar{k}_{1} \geq \cdots \geq \bar{k}$. Hence, the sequence $(\mathbf{c}, \mathbf{k})$ is feasible from $k^{*}$ in Problem $\mathcal{Q}$ with the convex technology represented by the production function $F$. Moreover, knowing that $k^{*}$ is an optimal steady state of Problem $\mathcal{Q}$, we have

$$
\sum_{t=0}^{\infty} \beta^{t} u\left(c^{*}\right) \geq \sum_{t=0}^{\infty} \beta^{t} u\left(c_{t}\right) .
$$


Since the set of sequences that is feasible from $\left(k^{*}, \bar{k}\right)$ for the problem $\mathcal{P}^{\prime}$ is a subset of the corresponding one for the Problem $\mathcal{Q}$, we can conclude that $\left(k^{*}, \bar{k}\right)$ is an optimal steady state for the original problem $\mathcal{P}^{\prime}$ with the technology $\tilde{f}$.

Now we will present our main result. The threshold will be exhausted in a finite period of time provided that the technology to reduce the fixed costs is sufficiently efficient $\left(g^{\prime}(0)<1\right.$, by Assumption 3) and the initial level of fixed costs is not too large $\left(\hat{k}\left(\bar{k}_{0}\right)<k^{s}\right.$, by Assumption 6$)$.

The idea of the proof is as follows. We suppose that the optimal steady state threshold $\bar{k}$ is strictly positive. We construct a feasible sequence $(c, k, \bar{k})$ starting from $k_{0}=k^{*}, \bar{k}_{0}=\bar{k}$. Since $\bar{k}>0$, to diminish the threshold by a small amount, say $\epsilon$, in any period $t \geq 1$, the economy invests $g(\epsilon)$ in period 0 . The consumption in period 0 becomes smaller than the steady state consumption, but the consumptions in other periods become higher. This proves that the consumer will be better off. That is a contradiction. The steady state threshold must be zero.

PROPOSITION 3: $\bar{k}=0$, i.e., the threshold will be exhausted in finite time.

Proof: First observe that $\bar{k}<k^{*}$. The optimal steady state consumption is

$$
c^{*}=f\left(k^{*}-\bar{k}\right)-\delta k^{*} .
$$

It is strictly positive under Assumption 5 . Assume $\bar{k}>0$. Then we can choose $0<\epsilon<$ $\bar{k}, g(\epsilon)<c^{*}$. Define the sequence $(\boldsymbol{c}, \boldsymbol{k}, \overline{\boldsymbol{k}})$ by

$$
\begin{aligned}
& \bar{k}_{0}=\bar{k}, \bar{k}_{t}=\bar{k}-\epsilon, \forall t \geq 1, \\
& k_{t}=k^{*}, \forall t \geq 0, \\
& c_{0}=f\left(k^{*}-\bar{k}\right)-\delta k^{*}-g(\epsilon), \\
& c_{t}=f\left(k^{*}-\bar{k}_{t}\right)-\delta k^{*}=f\left(k^{*}-\bar{k}+\epsilon\right)-\delta k^{*}, \forall t \geq 1 .
\end{aligned}
$$

Observe $c_{0}=c^{*}-g(\epsilon)>0, c_{t}>c^{*}>0, \forall t \geq 1$. One can easily check that the sequence $(\boldsymbol{c}, \boldsymbol{k}, \overline{\boldsymbol{k}})$ is feasible. Let

$$
\triangle(\epsilon)=\sum_{t=0}^{\infty} \beta^{t} u\left(c_{t}\right)-\sum_{t=0}^{\infty} \beta^{t} u\left(c^{*}\right) .
$$

We have

$$
\begin{aligned}
\triangle(\epsilon)= & u\left(f\left(k^{*}-\bar{k}\right)-\delta k^{*}-g(\epsilon)\right)+\frac{\beta}{1-\beta} u\left(f\left(k^{*}-\bar{k}+\epsilon\right)-\delta k^{*}\right) \\
& -u\left(f\left(k^{*}-\bar{k}\right)-\delta k^{*}\right)-\frac{\beta}{1-\beta} u\left(f\left(k^{*}-\bar{k}\right)-\delta k^{*}\right)
\end{aligned}
$$

that implies

$$
\begin{aligned}
\Delta(\epsilon) \geq & u^{\prime}\left(f\left(k^{*}-\bar{k}\right)-\delta k^{*}-g(\epsilon)\right)(-g(\epsilon)) \\
& +\frac{\beta}{1-\beta} u^{\prime}\left(f\left(k^{*}-\bar{k}+\epsilon\right)-\delta k^{*}\right)\left(f\left(k^{*}-\bar{k}+\epsilon\right)-f\left(k^{*}-\bar{k}\right)\right) .
\end{aligned}
$$


Hence,

$$
\begin{aligned}
\frac{\triangle(\epsilon)}{\epsilon} \geq & -\frac{g(\epsilon)}{\epsilon} u^{\prime}\left(f\left(k^{*}-\bar{k}\right)-\delta k^{*}-g(\epsilon)\right) \\
& +\frac{\beta}{1-\beta} u^{\prime}\left(f\left(k^{*}-\bar{k}+\epsilon\right)-\delta k^{*}\right)\left(\frac{f\left(k^{*}-\bar{k}+\epsilon\right)-f\left(k^{*}-\bar{k}\right)}{\epsilon}\right) .
\end{aligned}
$$

Let $\epsilon \rightarrow 0$. Then, note that

$$
\begin{aligned}
\lim _{\epsilon \rightarrow 0} \frac{\triangle(\epsilon)}{\epsilon} & \geq u^{\prime}\left(c^{*}\right)\left[-g^{\prime}(0)+\frac{\beta}{1-\beta} f^{\prime}\left(k^{*}-\bar{k}\right)\right] \\
& =u^{\prime}\left(c^{*}\right)\left[-g^{\prime}(0)+1+\frac{\beta \delta}{1-\beta}\right] \\
& >u^{\prime}\left(c^{*}\right) \frac{\beta \delta}{1-\beta}>0, \text { since } g^{\prime}(0)<1 .
\end{aligned}
$$

Thus, $\Delta(\epsilon)>0$ for $\epsilon$ small enough contradicting the optimality of the steady state. We conclude that $\bar{k}=0$.

We have proved that the threshold disappears within a finite period of time.

THEOREM 1: The optimal path $(\boldsymbol{k}, \overline{\boldsymbol{k}})$ from $\left(k_{0}, \bar{k}_{0}\right) \geq 0$ converges to $\left(k^{s}, 0\right)$.

Proof: By Proposition 3, the problem $\mathcal{P}^{\prime}$ actually reduces to the standard Ramsey model.

REMARK 1: In our model three assumptions are crucial for the fixed costs to disappear in finite time. The first one is the efficiency of the technology for reducing the fixed costs which is $\varphi^{\prime}(0)>1$. The two other assumptions, Assumptions 5 and 6, impose that the initial fixed costs are not very high. Actually, they give an upper bound to these initial fixed costs which is the same given the production technology. The economies which have the same technology of production and satisfy all our assumptions will eventually converge to the same steady state. Their technologies for reducing the fixed costs and their initial fixed costs may differ but they must satisfy the three crucial assumptions mentioned above.

\section{Conclusion}

We have considered an underdeveloped economy with nonconvexities in technology due to a wide variety of factors that induce fixed costs in production. We have proved that such an economy can avoid a poverty trap and catch up with the developing economies if its initial level of fixed costs are not too large and its technology for reducing the fixed cost is sufficiently efficient. We have shown that even though the income disparities may be very persistent and can be perceived as poverty traps, all economies with not very high initial fixed costs and sufficiently efficient technology to reduce fixed costs would ultimately converge to the same steady state level of per capita income. This induces a unimodal cross country income distribution where bimodality would appear during the transition.

The results in the present paper suggest that the aid policies aiming to help underdeveloped economies escape from a poverty trap should take into account not only 
the level of fixed costs inherent in production but also the efficiency of the technology to reduce them. Accordingly, apart from its size, the composition of the financial aids among the reduction of fixed costs, improving the technology for reducing these fixed costs, and fostering the accumulation of physical capital turn out to be crucial.

\section{References}

AKAO, K., T. KAMIHIGASHI, and K. NISHIMURA (2011) Monotonicity and continuity of the critical capital stock in the Dechert-Nishimura model, Journal of Mathematical Economics 47, 677-682.

AZARIADIS, C., and J. STACHURSKI (2004) A forward projection of the cross-country income distribution. Mimeo, Université Catholique de Louvain.

AZARIADIS, C., and J. STACHURSKI (2005) Poverty traps. In Handbook of Economic Growth, Vol. 1, P. Aghion and S. Durlauf, eds., pp. 295-384. Amsterdam: Elsevier.

DECHERT, W. D., and K. NISHIMURA (1983) A complete characterization of optimal growth paths in an aggregated model with non-concave production function, Journal of Economic Theory 31, 332-354.

DEMEKE, M., T. FREDE, B. ASSEFA, D. ALEMU, and M. ASSEFA (2004) Smallholder vegetable and pepper production in Ethiopia: A case study in Meki, Ziway, Awassa and Meskano areas, a research report prepared for the International Development Enterprise (IDE), Washington, DC.

FEYRER, J. (2008) Convergence by parts, The B.E. Journal of Macroeconomics 8(1), 1-35.

HANJRA, M. A., T. FEREDE, and D. G. GUTTA (2009) Pathways to breaking the poverty trap in Ethiopia: Investments in agricultural water, education, and markets, Agricultural Water Management 96(11), 2-11.

HUNG, N.M., C. LE VAN, and P. MICHEL (2009) Non-convex aggregate technology and optimal economic growth, Economic Theory 40, 457-471.

JONES, C. (1997), Convergence revisited, Journal of Economic Growth 2(2), 131-153.

KAMIHIGASHI, T., and S. ROY (2007) A nonsmooth, nonconvex model of optimal growth, Journal of Economic Theory 132, 435-460.

KRAAY, A., and C. RADDATZ (2007) Poverty traps, aid, and growth, Journal of Development Economics 82, 315-347.

KREMER, M., A. ONATSKI, and J. STOCK (2001) Searching for prosperity, Carnegie-Rochester Conference Series on Public Policy 55(1), 275-303.

LE VAN, C., and L. MORHAIM (2002) Optimal growth models with bounded or unbounded returns: A unifying approach, Journal of Economic Theory 105, 158-187.

MITRA, T., and D. RAY (1984) Dynamic optimization on a non-convex feasible set: Some general results for non-smooth technologies, Journal of Economics 44, 151-175.

ROSEGRANT, M. W., C. RINGLER, T. BENSON, X. DIAO, D. RESNICK, J. THURLOW, M. TORERO, and D. ORDEN (2006) Agriculture and Achieving the Millenium Development Goals. Washington, DC: The World Bank (Agriculture and Rural Development Department).

QUAH, D. T. (1996) Convergence empirics across economies with (some) capital mobility, Journal of Economic Growth 1, 95-124.

QUAH, D. T. (1997) Empirics for growth and distribution: Stratification, polarization, and convergence clubs, Journal of Economic Growth 2, 27-59.

QUAH, D. T. (2001) Searching for prosperity: A comment, Carnegie-Rochester Conference Series on Public Policy 55(1), 305-319.

WORLD BANK (1994) World Development Report: Infrastructure for Development. New York: Oxford University Press.

YOSHINO, Y. (2008) Domestic constraints, firm characteristics, and geographical diversification of firm-level manufacturing exports in Africa. Policy Research Working Paper No. 4575, World Bank, Washington, DC. 